\title{
Urinary Tract Infection, Incidence, Uropathogens and Antibiotic Sensitivity in Females
}

\author{
Mahaseth $\mathrm{BK}^{1}$, Verma $\mathrm{P}^{2}$, Malla $\mathrm{T}^{3}$
}

\begin{abstract}
Introduction: Urinary tract infection in female population is one of the most common clinical infectious pathologies found worldwide due to anatomical location of urethral opening in relation to males. Continuous assessment of uropathogens and their sensitiveness to chemotherapeutic agent help us to manage these. The aim of this study to determine the presence of uropathpgens and their sensitkng from UTI. Method: It is a hospital based cross sectional study conducted at NGMC Teaching Hospital Kohalpur from July 2016 to June 2017. The urine samples were collected using the mid-stream clean catch method from 948 clinically suspected UTI female patients and the irantibiotic sensitiveness was determined using the standard procedures. Result: Overall culture positive was found in 262 patients (27.6\%) among 948 urine samples. Among them e. coli contributed to cases 179(68.3\%) followed by klebsiella $38(14.5 \%)$, enterobacter $21(8 \%)$, fungal $10(3.9 \%)$. Maximum no. of uropathogens were sensitive to chloramphenicol 224(93.4\%) followed by nitrofurantin 232(88.7\%), amikacin 246(94\%), tobramycin $241(92.8 \%$ ) and gentamycin 229(85.5\%). Most of the organism were found to be resistant with ampicillin 255(97.5\%) followed by cefpodoxime 242(92.4\%), vancomycin 235(90\%) and amoxicillin 234(89.6). Conclusion: and recommendation - Chloramphenicol, Nitrofurantin, Amikacin, Gentamicin and Tobramycinare the drugs of choice for the empirical therapytill the culture and sensitivity report is available. Continuous assessment is required for the early diagnosis and management of UTI.The culture and the sensitiveness of uropathogen are to be done and the treatment should be modified accordingly.
\end{abstract}

Key words: Antibiotic, UTI, uropathogen, sensitivity, resistant

\section{INTRODUCTION}

Urinary tract infection (UTI) is one of the most common clinical entity encounters with community practice. It accounts for the high rate of morbidity and financial burden worldwide. It is estimated that 150 million people are infected with UTI per annum worldwide. This makes global economy burden of more than 6 billion US dollars in a year ${ }^{1}$. UTI describes with presence of bacteriurea with clinical symptom, like dysuria, frequency, urgency, suprapubic pain, renal angle pain or flanks pain and sometimes with fever, nausea and vomiting. UTI can be classified as lower and upper, according to anatomical site of presence of infection.

Anatomical classification of UTI divides into upper and lower, when kidney and ureter were involved with pathological process, known as upper UTI, if pathology involving urinary bladder and urethra known as lowers UTI. However, we need to remember that if lower urinary tract is involved it does not mean upper tract may be free or vice versa. Most of the cases of lower UTI involvement is known as a cystitis, presented with

\footnotetext{
1. Dr. Binod Kumar Mahaseth

2. Mr. Pramod Verma

3. Mr. Tark Malla
}

Address for correspondence: Dr. Binod Kumar Mahaseth Department of Obst. \& Gynae. Nepalgunj Medical College Teaching Hospital Kohalpur, Nepal Email: mahaseth.binod@yahoo.com symptom likedysuria, frequency, urgency, suprapubic pain with bacteriurea ${ }^{2,3}$. Depending on choice of treatment, UTI is classified in to complicated and non-complicated ${ }^{4}$.

Incidence of UTI is more common in females compared to male due to short urethra in females and structurally found less effective in preventing the bacterial entry due to the proximity of the genital tract and urethra, adherence of urothelial mucosa to the mucopolysaccharide lining, pregnancy and sexual activity.

Trienekens TAM et al, reported an overall incidence of $50 / 1000 /$ year $^{6}$. In uncomplicated cases mostly treated with empirical antibiotic without knowing uropathogen and its sensitiveness, it's also contributes to make microflora resistant to mostly used antibiotics ${ }^{7}$. Overall estimated that in one third of women treated for UTI at least one time by the age of 26 years, its incidence increases to approximately $60 \%$ during a woman's lifetime, signifies burden of disease in female population ${ }^{8,9}$.

Clinicians agree regarding local in vitro resistance rates are not always known, even known changing pattern regarding sensitiveness strain of microorganism and changing of uropathogen over a time is anticipated, thinking of this continuous assessment of uropathogen identification is necessary to manage a infective morbidities for local population. We are living in low income country where cost makes value of purposeless use of antibiotic, a major burden cost wise even leads resistant to microflora and required newer 
and newer antimicrobial agent to manage these patients, makes burden to Pharma Company to introduce newer antibiotic. Thinking of this given study can helps health professional to carry out choice for antibiotic selection in empirical therapy to manage uncomplicated UTI cases and further helps to make guidelines to manage UTI related cases.

\section{MATERIALAND METHOD}

The present study was carried out in the department of Gyn/obsNepalgunj Medical College Teaching Hospital, Kohalpur. It is a cross sectional study consisting of 948 patients admitted during July 2016 to July 2017.Mid-stream urine sample collected who came with complain of like dysuria, frequency, urgency, suprapubic pain, renal angle pain or flanks pain with occasional fever, nausea and vomiting and not treated with antibiotic for at least two weeks prior to inclusion in the study, group were included excluded in study. Patients who were already getting treatments for UTI, diagnosed cases of immunosuppressive, diabetics and who refused to give urine samples, were excluded from the study.

\section{AIMS AND OBJECTIVE}

To determine the

- Overall presence of uropathogen causing UTI.

- Type of organism causing UTI.

- Antibiotic sensitiveness with common using antibiotic for identified uropathogen.

- $\quad$ Antibiotic resistantwith common using antibiotic for identified uropathogen.

Samples were collected in sterile container and processed in microbiology lab.as standered procedures by using biochemical tests including catalase, coagulase, oxidase, indole, methyl red, citrate, urease, triple sugar, iron agar and motility.

\section{RESULT}

In present study $628(76.8 \%)$ cases of clinically suspected UTI fall in the age group of $10-50$ years. this may be noted that 20 years and above are from the reproductive age group. $h$

Mean age $32+-15.7$ yrs of age and minimum age is 15 and maximum age 85 yrs (Table I).

\begin{tabular}{|c|c|c|}
\hline Age (Years) & Frequency & Percentage \\
\hline $10-20$ Yrs & 75 & 7.9 \\
\hline $20-30$ & 345 & 36.4 \\
\hline $30-40$ & 196 & 20.7 \\
\hline $40-50$ & 112 & 11.8 \\
\hline >= 50 Yrs & 220 & 23.2 \\
\hline Total & 948 & 100 \\
\hline
\end{tabular}

Table I: Age distribution of study participants
Total case selected for study purpose was 973, among them $25(2.6 \%)$ cases were excluded due to refusal in participation. Out of 948 samples 686(72.4\%) samples were negative for growth and $262(27.6 \%)$ samples grew bacteria such samples were further investigated for culture and sensitivity. Out of 262 samples most common bacteria was E. coli 179 cases $(68.3 \%)$ which was followed by klebsiella 38 cases $(14.5 \%)$, enterobacter 21 cases (8\%), fungal 10 cases (3.9\%), acinetobacter 6 cases (2.3\%)pseudomonas 4 cases $(1.5 \%)$ staph in 1 case $(0.4 \%)$ (Table II).

\begin{tabular}{|c|c|c|}
\hline Uropathogens & Frequency & Percentage \\
\hline E.coli & 179 & 68.3 \\
\hline Klebsiella & 38 & 14.5 \\
\hline Enterobacte & 21 & 8.0 \\
\hline Fungal & 8 & 3.1 \\
\hline Acinetobacter & 6 & 2.3 \\
\hline Pseudomonas & 4 & 1.5 \\
\hline Proteus & 3 & 1.1 \\
\hline Candida & 2 & 0.8 \\
\hline Staph & 1 & 0.4 \\
\hline Total & 262 & 100 \\
\hline
\end{tabular}

Table II: Incidence of bacterial uropathogens UTI suspected female patients

Grown organism were tested for sensitiveness. Maximum no. of uropathogen were sensitive with chloramphenicol (c) 224(93.4\%) in 241 samples followed bynitrofurantin (Nit)232 (88.7\%), amikacin (AK) 246(94\%), tobramycin (tob) 241(92.8\%) and gentamycin (gen) 229(87.5\%) samples. Most of the organism were resistant with ampicillin 255(97.5\%) followed bycefpodoxime (CPD) 242(92.4\%), vancomycin 235(90\%) and amoxicillin 234(89.6) samples tested with antibiotic respectively.

\section{DISCUSSION}

In our study the urine culture were done on all patients who were never catheterised. In our study E Coli was found to be responsible in $68.3 \%$ of the cases. In the study conducted by Muhammad et $\mathrm{al}^{10}$, reported incidence of $\mathrm{E}$. coli as uropathogen in $67 \%$ cases (his patients were also not catheterised before the urine culture was sent). In our study klebsiella was the $2^{\text {nd }}$ most commonest offender. In Muhammad series pseudomonas was the second most common uropathogen causing UTI. The difference in the spectrum of uropathogens may be because of the geographical changes. In our series as wellas in Mohammad seroes Gentamycin was found to most efficient antiboitic. However, our cases were resistant to cotrimoxazole/trimethoprim in contrast to his series where cotrimoxazole was found to be second drug of choice.

Chih-C C et al ${ }^{11}$ also reported E.coli responsible for $54.5 \%$ cases 
of UTI and klebsiella was the $2^{\text {nd }}$ most offender constituting about $13.1 \%$ cases. He reported Amikacin and Gentamycin as the drug of choice. The sensitivity of uropathogen in the present series also confirms that Gentamycin was most effective.

Kahlmeter et $\mathrm{al}^{13}$ et al reported in multicountry study that e.coli as causative organism contributes $77 \%$ in present study $68.3 \%$ is comparable and reported most of the pathogen resistant with ampicillin comparable with present study ${ }^{13}$.

David et $\mathrm{al}^{14}$ found in his series that most of the uropathogens were E.coli (77.5\%) and Nitrofurantoin was the drug of choice. In a study carried out by Acharya et al in 2009 reported this in his series only $24.94 \%$ were positive for culture. In our cases urine culture was positive in $27.6 \%$ of the cases who came with the symptoms of UTI.

Study conducted by Joshi et al again reported most common organism E.coli as the commonest uropathogen (66.7\%). In his series the overall culture was positive in around $25.24 \%$.

\section{CONCLUSION}

1. The female patients admitted to the hospital with the symptoms of UTI (i.e. dysuria, frequency, urgency, suprapubic pain, renal angle pain or flanks pain with occassional fever, nausea and vomiting), surprisingly show presence of bacteria only in $25-30 \%$ of the cases (in the present series $27.6 \%$ ).

2. E. coli is the most commonest uropathogen causing UTI $68.3 \%$ cases. Second commonest offender was klebsela in our series

3. Gentimycin was found to be most effective antibiotic against the organisms. However, in our series Cotrimoxazole was found to be ineffective due to the growth of the resistant strains.

\section{LIMITATION OF THE STUDY}

Cost of culture is an economic burden and therefore poor patients are empirically treated with antibiotic prior to culture in our part of the world. The facilities of the urine culture must be made available to all patients. However, in absence of culture facilities if UTI is suspected the patients are recommended to receive Gentimycin on the basis of our study. Cotrimoxazole appears to be a poor choice if used emperically.

\section{ACKNOWLEDGMENTS}

I Thank Dr. Ram Das, Dr. Jyoti Lamichhane, department of Gyn/Obs, and Manoj Sah, Saket Bihari Sharma (Microbiology) and others who helped to carry out the present study.

\section{REFERENCES}

1. C. M. Gonzalez and A. J. Schaeffer, "Treatment of urinary tract infection: what's old, what's new, and what works," World Journal of Urology, vol. 17, no. 6, pp. 372-382, 1999.

2. I. Zelikovic, R. D. Adelman, and P. A. Nancarrow, "Urinary tract infections in children-an update," Western Journal of Medicine, vol. 157, no. 5, pp. 554-561, 1992.

3. J. D. Sobel and D. Kaye, "Urinary tract infections," in Mandell, Douglas and Bennett's Principles and Practice of Infectious Diseases, G. L. Mandell, J. E. Bennett, and R. Dolin, Eds., pp. 957-985, Churchill Livingstone, Philadelphia, Pa, USA, 7th edition, 2010.

4. S. M. Sabra and M. M. Abdel-Fattah, "Epidemiological and microbiological profile of nosocomial infection in Taif hospitals, KSA (2010-2011)," World Journal of Medical Sciences, vol. 7, no. 1, pp. 1-9, 2012.

5. J. W. Warren, E. Abrutyn, J. Richard Hebel, J. R. Johnson, A. J. Schaeffer, and W. E. Stamm, "Guidelines for antimicrobial treatment of uncomplicated acute bacterial cystitis and acute pyelonephritis in women," Clinical Infectious Diseases, vol. 29, no. 4, pp. 745-758, 1999.

6. Trienekens TAM, Stobberingh EE, Winkens RAG et al. Different lengths of treatment with cotrimoxazole for acute uncomplicated urinary tract infections in women. Br Med J 1989; 299: 1319-22

7. Hillier $S$, Bell J, Heginbothom $M$ et al. When do general practitioners request urine specimens for microbiology analysis? The applicability of antibiotic resistance surveillance based on routinely collected data. J AntimicrobChemother 2006; 58: 1303-6.

8. Foxman B, Barlow R, D'Arcy H, Gillespie B, Sobel JD. Urinary tract infection: selfreported incidence and associated costs. Ann Epidemiol. 2000;10:509-515.

9. Hooton TM, Besser R, Foxman B, Fritsche TR, Nicolle LE. Acute uncomplicated cystitis in an era of increasing antibiotic resistance: a proposed approach to empirical therapy. Clin Infect Dis. 2004;39:75-80.

10. Muhammad D H, SunjuktaA, Md. S K.Antibiotic resistance patterns of uropathogens isolated from catheterized and noncatheterized patients in Dhaka, Bangladesh.Tzu Chi Medical Journal2014: $26 ; 127-131$

11. Chih-C C, Tzu-C L, Rui-Xin W , Ya-S Yb , Po-J H, Yi L, Jung-C L, Feng-Y C.Etiologies of community-onset urinary tract infections requiring hospitalization and antimicrobial susceptibilities of causative microorganisms.Journal of Microbiology, Immunology and Infection 2017: 50; 879-885

12. Mahmoud A. M, Tarig M.S., Osama M. S , Mariam M. A.Prevalence and antimicrobial resistance pattern of bacterial strains isolated from patients with urinary tract infection in Messalata Central Hospital, Libya.Asian Pacific Journal of Tropical Medicine 2016; 9(8): 771-776

13. KahlmeterG.An international survey of the antimicrobial susceptibility of pathogens from uncomplicated urinary tract infections: the ECO.SENS Project. Journal of Antimicrobial Chemotherapy 2003: 51; 69-76.

14. David D B, Thierry C, Stefan H, SutterA D, Ellen E. S and Verschraegen G.Evolution of bacterial susceptibility pattern of Escherichia coli in uncomplicated urinary tract infections in a country with high antibiotic consumption: a comparison of two 
surveys with a 10 year interval.Journal of Antimicrobial Chemotherapy 2008:62; 364-368.

15. AcharyaA, Gautam $R$ and Subedee.Uropathogens and their antimicrobial susceptibility pattern in Bharatpur, Nepal.Nepal Med Coll J 2011; 13(1): 30-33.

16. Joshi Y P, Shrestha S, Kabir R, Thapa A, Upreti P, ShresthaS.Urinary tract infections and antibiotic susceptibility among the patients attending B \& D hospital of Lalitpur, Nepal.Asian Journal of Medical Sciences 2016: 7 (5); 47-51. 